\title{
Sentinel Lymph Nodes Show Profound Downregulation of Antigen-Presenting Cells of the Paracortex: Implications for Tumor Biology and Treatment
}

\author{
Alistair J. Cochran, M.D., Donald L. Morton, M.D., Stacey Stern, Ph.D., Ana M.A. Lana, M.D., \\ R. Essner, M.D., Duan-Ren Wen, M.D. \\ Departments of Pathology and Laboratory Medicine and Surgery and Jonsson Comprehensive Cancer \\ Center, University of California Los Angeles, School of Medicine, Los Angeles, California (AJC, D-RW); the \\ John Wayne Cancer Institute, Saint John's Hospital and Health Center, Santa Monica, California (DLM, \\ SS, RE); and Departamento de Anatomia Pathológica, Faculdade de Medicina da Universidade Federal de \\ Minas Gerais, Belo Horizonte, Minas Gerais, Brazil (AMAL)
}

The sentinel lymph node (SN) is the first node on the direct lymphatic drainage pathway from a tumor. Melanoma-associated SNs are the most likely site of early metastases and their immune functions are strikingly down-modulated. We evaluated histologic and cytologic characteristics of 21 SNs and 21 nonsentinel nodes (NSNs) from melanoma patients who had clinically localized (AJCC Stage I-II) primary cutaneous melanoma. SNs showed highly significant reductions in total paracortical area and in the area of the paracortical subsector occupied by dendritic cells. The frequency of paracortical interdigitating dendritic cells (IDCs) was dramatically reduced in SNs, and most IDCs $(\sim 99 \%)$ lacked the complex dendrites associated with active antigen presentation. The release of immunosuppressive factors from the primary melanoma may induce a localized and specific paralysis in the $\mathrm{SN}$, which prevents the recognition of otherwise immunogenic melanoma antigens by IDCs. This immune paralysis may facilitate the implantation and growth of melanoma cells in the SN. Cytokine therapy may be able to reverse this immune paralysis. These findings have an important practical application in the histopathologic confirmation that a node is truly sentinel. They also offer an hypothesis to explain the failure of the immune surveillance mechanisms to identify and respond to a small primary melanoma that expresses immunogenic tumor antigens.

Copyright () 2001 by The United States and Canadian Academy of Pathology, Inc.

VOL. 14, NO. 6, P. 604, 2001 Printed in the U.S.A.

Date of acceptance: February 14, 2001.

Address reprint requests to: Alistair J. Cochran, M.D., Department of Pathology and Laboratory Medicine, UCLA School of Medicine, Los Angeles, CA 90095-1713; e-mail: acochran@mednet.ucla.edu.
KEY WORDS: Dendritic leukocytes, Melanoma, Paracortex, Sentinel lymph nodes.

Mod Pathol 2001;14(6):604-608

Regional metastasis of a small primary cutaneous melanoma defies the precepts of tumor immunology. A melanoma that is only a few millimeters in diameter or thickness but that expresses multiple immunogenic tumor antigens should be recognized by dendritic cells, triggering a T lymphocyte-mediated rejection response. From extensive studies of regional lymph nodes draining primary melanomas and breast cancers (1-15), we hypothesize that the antitumor immune functions of lymph nodes close to the tumor are down-regulated by tumor-derived products.

We previously reported that some lymph nodes regional to melanoma and breast cancer are immune suppressed and that the degree of immune suppression correlates directly with the closeness of the node to the tumor (1). Nodes proximal to the tumor or partly replaced by tumor have reduced paracortical activity. They also have fewer interdigitating dendritic cells (IDCs) (2). IDCs are dendritic leukocytes in the T-cell-rich paracortex of the lymph node that act as highly specialized accessory cells in the induction of immunity and tolerance (16). IDCs acquire, process, and present antigens; bind and stimulate infrequent antigen-specific T-cell clones; and generate immunity within the whole animal (16). In nodes located near to tumor, IDCs lack the complex dendrites that characterize active antigen presentation and lack markers of activation such as HLA-Dr.

We have developed the techniques of lymphatic mapping and sentinel lymphadenectomy (LM/SL) $(1,7)$, which permit studies of lymph nodes on the direct lymphatic pathway from the tumor. The re- 
gional node basin that receives lymph from a primary melanoma is identified by lymphoscintigraphy, and the first node in that basin to receive lymph from the primary tumor area, the sentinel node ( $\mathrm{SN}$ ), is identified by reading the lymphoscintigram 20-30 minutes after injection of contrast medium. The site of the SN is confirmed at the time of operation by injecting an inert blue dye (isosulfan blue) and a radiolabeled marker intradermally at the primary tumor site at the beginning of the procedure. The surgeon identifies the SN by following blue-stained lymphatics to one or more bluestained nodes that exhibit increased radioactivity when scanned with a handheld gamma probe.

Following the method of previous observations of variations in dendritic cell distribution and phenotype in lymph nodes close to a tumor (2), we studied and compared dendritic cells in SNs and nonsentinel nodes (NSNs) from the same patient. This class of dendritic cell is confined to the paracortex, and IDCs are strikingly polydendritic when presenting antigen to client lymphocytes. They can be readily visualized by immunocytochemical staining using markers such as MHC class II antigens, S-100 protein, and CD1a.

We found a profound and highly selective downregulation of IDCs in SNs compared with non-SNs (NSNs). We postulate that this down-regulation paralyzes the immune response to immunogenic melanoma antigens released by the primary melanoma.

\section{MATERIALS AND METHODS}

Blocks of nodal tissue from 11 patients with clinically localized (AJCC Stages I and II) primary cutaneous melanoma were retrieved from pathology files at the UCLA School of Medicine and at the John Wayne Cancer Institute of Saint John's Health Center. All patients had undergone LM/SL followed by complete lymphadenectomy, during which a total of 21 SNs and 226 NSNs were excised. We matched each SN with a comparably sized NSN from the same patient. Ten SNs contained micro- metastatic melanoma and 11 were tumor free. All NSNs were tumor free. These 21 matched pairs were examined as described below.

Four-micrometer sections were cut from formalinfixed, paraffin-embedded tissue blocks. Standard labeled avidin-biotin (LAB) immunoperoxidase staining was performed (17). Monoclonal antibodies CD43 and CD20 were obtained from DAKO Corporation (Carpinteria, CA) and used to identify $\mathrm{T}$ cells and $\mathrm{B}$ cells, respectively. The polyclonal antibody against S-100 protein, used to identify IDCs, was made in our laboratory.

Slides were analyzed using an IBM PC-assisted image analysis system (planar morphometry, Version 2.1, Southern Micro Instruments, Atlanta, GA). Each SN and matched NSN was measured for total T-cell area, total B-cell area, total area occupied by IDCs, density of IDCs in the four fields that contained the most IDCs, and frequency and length of IDC dendrites in these four fields. Areas were measured at $100 \times$ magnification; IDC density and dendritic processes were evaluated at $200 \times$ magnification.

Morphometric data were analyzed using a statistical package inbuilt to the image analysis system. Comparisons between SNs and NSNs were made using the Wilcoxon signed-rank test and twosample $t$ tests with equal variance.

\section{RESULTS}

\section{Paracortical, IDC, and B-Cell Areas}

Paracortical areas were identified by measuring the area occupied by cells that express CD43. The mean paracortical area was significantly smaller in SNs than in NSNs $(P<.0001$; Table 1; Fig. 1), regardless of whether or not the SN contained tumor. The area of the paracortex occupied by dendritic cells (IDC area), identified by expression of S-100 protein, also was significantly smaller in SNs than in NSNs $(P<.0001$; Table 1$)$. B lymphocytes in the lymph nodes were identified by expression of the CD20 marker. The mean area occupied by B

TABLE 1. Paracortical Area, IDC Area, and B-Cell Area in Sentinel (SN) and Nonsentinel Nodes (NSN)

\begin{tabular}{|c|c|c|c|c|}
\hline Sector & $\begin{array}{l}\text { Number } \\
\text { of Nodes }\end{array}$ & $\begin{array}{c}\text { Mean } \pm \text { SEM } \\
(\%)\end{array}$ & $\begin{array}{c}\text { Range } \\
(\%)\end{array}$ & $\begin{array}{c}P \text { Value } \\
(\mathrm{SN} \text { versus } \mathrm{NSN})\end{array}$ \\
\hline \multicolumn{5}{|c|}{ Paracortical area $\left(\mathrm{CD} 43^{+}\right)$} \\
\hline $\mathrm{SN}$ & 21 & $11.4 \pm 2.6$ & $00.18-29.4$ & $<0.0001$ \\
\hline NSN & 21 & $31.0 \pm 12.9$ & $10.60-70.0$ & \\
\hline \multicolumn{5}{|l|}{ IDC area $\left(\mathrm{S}_{100^{+}}\right)$} \\
\hline $\mathrm{SN}$ & 21 & $2.0 \pm 0.68$ & $0.00-10.2$ & $<0.0001$ \\
\hline NSN & 21 & $23.5 \pm 1.4$ & $1.50-47.7$ & \\
\hline \multicolumn{5}{|c|}{ B-Cell area $\left(\mathrm{CD} 20^{+}\right)$} \\
\hline $\mathrm{SN}$ & 21 & $24.0 \pm 2.1$ & $4.3-34.2$ & 0.1467 \\
\hline NSN & 21 & $28.5 \pm 1.4$ & $5.4-69.0$ & \\
\hline
\end{tabular}

IDC, interdigitating dendritic cells.

Values expressed as percentage of total nodal area. 


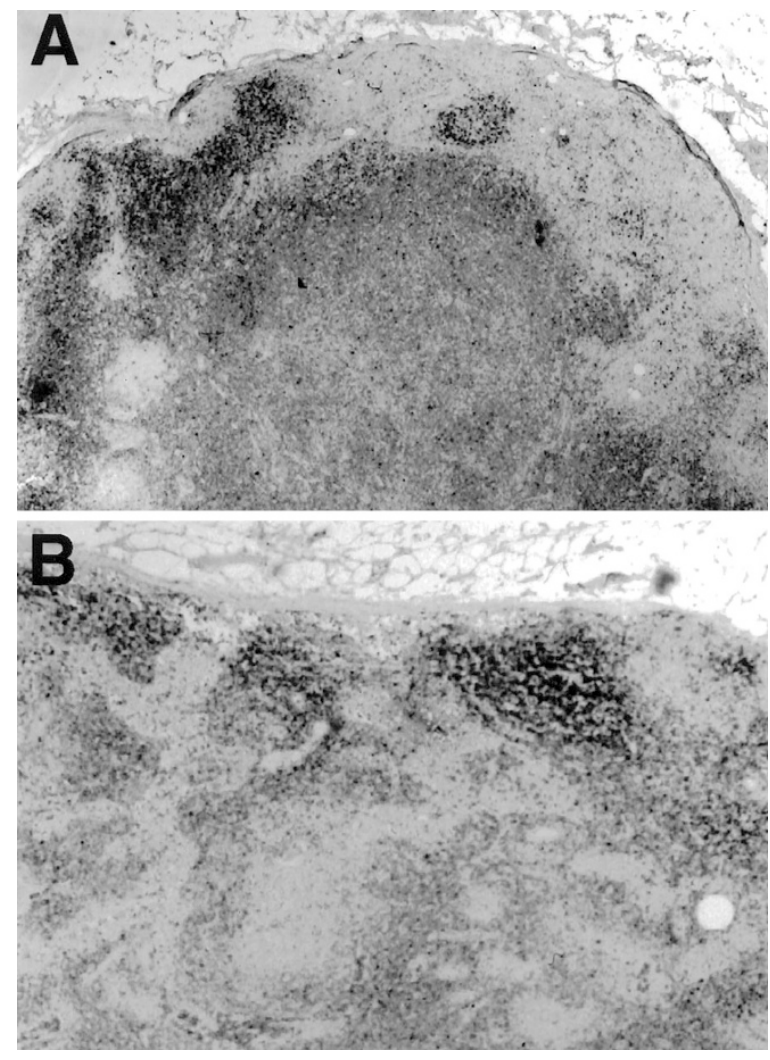

FIGURE 1. Showing the relative area of a sentinel node (B) and nonsentinel node (A) occupied by the paracortex. (Antibody to CD43, original magnification $40 \times$.)

lymphocytes was not significantly different in SNs and NSNs $(P=.1467$; Table 1$)$.

\section{IDC Density}

IDCs were identified by expression of the S-100 protein marker. Maximum IDC density was significantly lower in SNs than NSNs $(P<.0001$; Table 2; Fig. 2). Of the 21 matched pairs of SNs and NSNs, 11 SNs but no NSNs contained tumor. There was a significant difference in IDC area and IDC density between tumor-free SNs and matched tumor-free
NSNs and between tumor-positive SNs and matched tumor-free NSNs $(P<.01$ in each case; Table 2).

\section{Frequency and Length of the Dendritic Processes of IDCs}

Most IDCs in SNs either lacked dendrites or had short dendrites (Table 3; Fig. 2). Less than 1\% of IDCs in SNs had long dendrites. In contrast, only $36.99 \pm 1.18 \%$ of IDCs in NSNs lacked dendrites $(P$ $=.0001)$, and $23.4 \pm 1.13 \%$ of IDCs in NSNs had long dendrites $(P=.0001)$. There was no statistical difference between SNs and NSNs with respect to the frequency of IDCs with short dendrites $(P=$ .1910; Table 3). This difference in IDC morphology between SNs and NSNs was independent of the tumor status of the SN (data not shown).

\section{DISCUSSION}

A link between proximal node immune suppression and early metastases is suggested by increased numbers of suppressor cells in nodes close to tumor $(8,9)$, alterations in the phenotype of lymphocytes from nodes near tumor (10), and reduced cytostatic activity of cells from nodes close to melanoma (11). Tumor-derived factors, including gangliosides (12) and prostaglandins (13), have been shown to modulate (nodal) lymphocyte and macrophage function (15). Tumor-induced immune suppression that facilitates metastasis has also been reported in squamous carcinoma of the head and neck (18).

Before the development of lymphatic mapping (7), it was not possible to identify the node(s) most influenced by tumor, in other words, the first node on the direct lymphatic pathway from a primary melanoma. We can now divide regional nodes into SNs, which certainly drain lymph from the area of a cutaneous melanoma, and NSNs, nodes which are

TABLE 2. IDC Area and Density of Pairs of Sentinel (SN) and Nonsentinel Nodes (NSN)

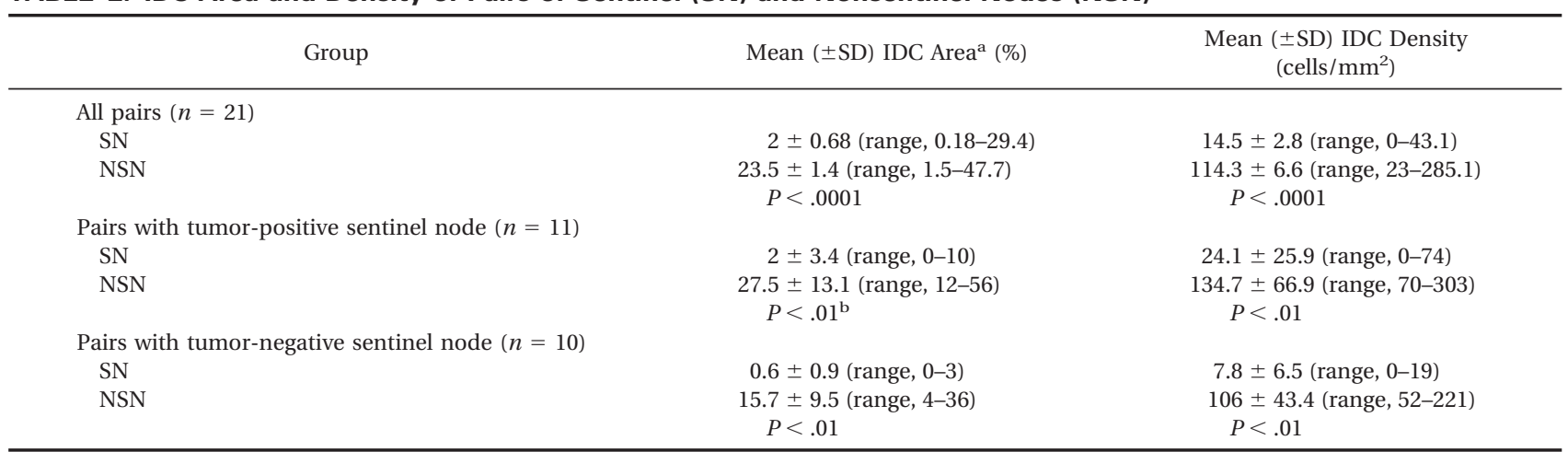

IDC, interdigitating dendritic cells.

${ }^{a}$ Percentage of total nodal area.

${ }^{\mathrm{b}}$ All statistical data derived from two-tailed critical values table for Wilcoxon signed-rank test. 


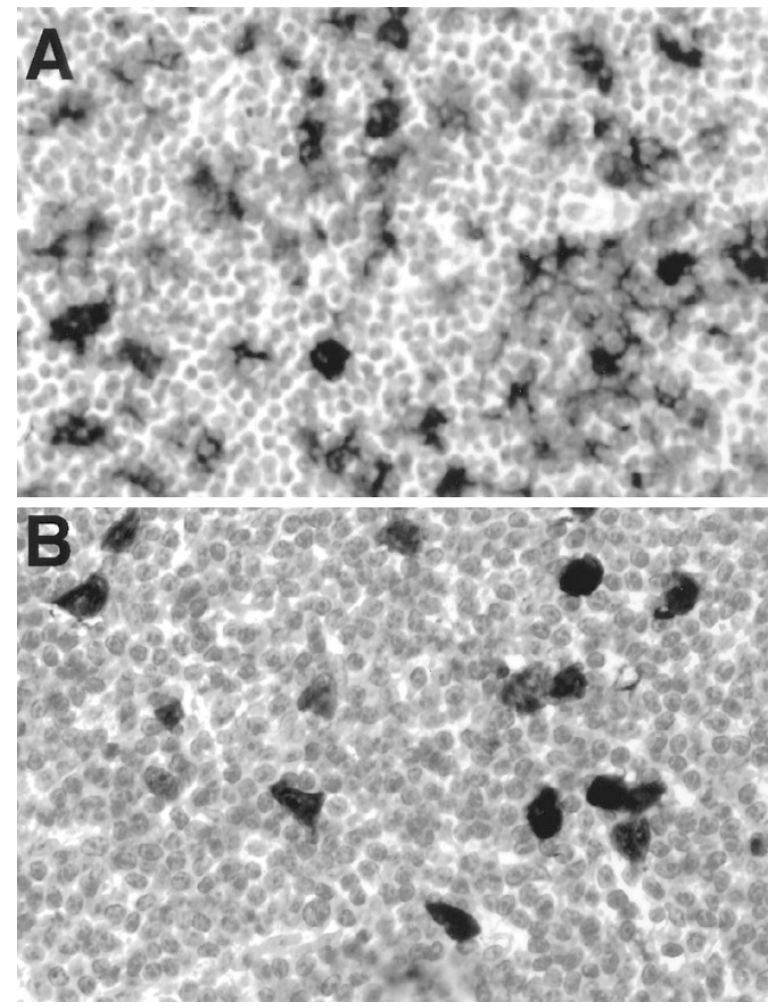

FIGURE 2. Showing dendritic cell populations in the sentinel node (B) and nonsentinel node (A). In the sentinel node, the number of dendritic cells is reduced, and they show little or no formation of dendritic processes. In contrast, in the nonsentinel node, dendritic cells are relatively abundant, and they show, for the most part, a complex, polydendritic morphology. (Polyclonal antibody to S-100 protein, original magnification, $400 \times$.)

more remote from the primary tumor or which may drain lymph from other areas of skin. NSN are likely to be less exposed to tumor-derived immunemodulating molecules. This allows us to test our hypothesis of selective immune suppression of tumor-proximate nodes. The data support the view that nodal immune suppression is induced by the primary tumor. Because the SNs are maximally immune suppressed, the selective occurrence of metastases in these nodes may be related to their immune down-regulation.

SN histology and cytology were markedly modified relative to NSNs. There was a major reduction in the total paracortical area of the node, the area and density of IDCs in the node, and the frequency of IDCs that had the complex (long) dendrites associated with active antigen presentation. This se- lective immunosuppressive effect on the paracortex and IDCs of SNs from melanoma patients is similar to alterations that we have observed in SNs removed from patients with breast cancer (14).

A highly focused and profound immune suppression of the SN might explain how a primary melanoma of only $1 \mathrm{~mm}$ thickness, but expressing immunogenic tumor antigens, can escape immune rejection and extend to establish regional nodal metastases. Normal processes by which tumorassociated antigens are presented to generate epitope-specific clones of $\mathrm{T}$ lymphocytes, including cytotoxic cells, are down-regulated. We envision a loop in which tumor-related Langerhans cells acquire tumor-associated antigens and migrate to the paracortex of regional nodes. After interactions with naive $\mathrm{T}$ lymphocytes in the paracortex, new clones of specifically sensitized $\mathrm{T}$ lymphocytes recirculate to the primary tumor, where they limit local extension of tumor and inhibit metastasis. If antigen presentation is arrested, as might follow a failure to recruit antigen-primed IDC to the SN or their down-regulation in the SN, generation of specifically cytotoxic $\mathrm{T}$ lymphocytes will diminish, favoring tumor growth, extension, and metastasis. Selective suppression of antigen presentation in SNs may be key to local progression of and regional metastasis from melanoma.

In addition to the biological significance of these findings, there is a potential practical application. Identification of the SN is currently based on lymphoscintigraphic localization and on observational (colorimetric) and gamma probe-based confirmation of a node's sentinel status by the surgeon. The pathologist records blue coloration in nodes as evidence in support of sentinel status, but the blue color often dissipates before a node is received in the laboratory. From our data, it may eventually be possible to identify a node as sentinel on the basis of reductions in paracortical area, IDC area, and the proportion of IDCs that express long dendritic processes. To allow practical day-to-day application of this approach, it will be necessary to develop technically simpler approaches.

Nodal immune suppression is due to tumor influence, mediated in part by melanoma-derived materials: gangliosides, prostaglandins, lipoprotein antigens, and cytokines transmitted via the lym-

TABLE 3. Distribution of IDCs with Long, Short, and Absent Dendrites in Sentinel Nodes and Nonsentinel Nodes

\begin{tabular}{|c|c|c|c|}
\hline \multirow{2}{*}{ Type of Lymph Node } & \multicolumn{3}{|c|}{ Mean \pm SE IDCs with (\%) } \\
\hline & Long Dendrites & Short Dendrites & No Dendrites \\
\hline Sentinel $(n=21)$ & $0.94 \pm 0.67$ (range, $0-10)$ & $33.0 \pm 4.72$ (range, $0-52.4)$ & $66.02 \pm 4.73($ range, $47.6-100)$ \\
\hline Nonsentinel $(n=21)$ & $\begin{aligned} 23.4 & \pm 1.13 \text { (range, } 0-40.7) \\
P & =.0001\end{aligned}$ & $\begin{aligned} 39.57 & \pm 0.86 \text { (range, } 21.8-64.3) \\
P & =.1910\end{aligned}$ & $\begin{aligned} 36.99 & \pm 1.18 \text { (range, } 10-65.3) \\
P & =.0001\end{aligned}$ \\
\hline
\end{tabular}

IDC, interdigitating dendritic cells. 
phatics from a primary tumor to the lymph nodes (1). Studies in progress will help identify the immune modulatory cytokines involved, the cells that generate these molecules, and their mechanisms of action. This may permit strategies to prevent or reverse immune suppression of tumor-related nodes and possibly reestablish an effective immune response against immunogenic melanoma antigens. Prevention or reversal of early metastases may thus be an attainable goal.

Acknowledgments: These studies were conducted with the assistance of funds from the National Cancer Institute (CA 29605). The authors thank Dr. Z. Farzad for making the polyclonal antibody against S-100 protein.

\section{REFERENCES}

1. Cochran AJ, Wen D-R, Farzad Z, Stene MA, McBride W, Lana $\mathrm{AM}$, et al. Immune suppression by melanoma cells as a factor in the generation of metastatic disease. Anticancer Res 1989;9:859-64.

2. Cochran AJ, Pihl E, Wen DR, Hoon DSB, Korn EL. Zoned immune suppression of lymph nodes draining malignant melanoma: histologic and immunohistologic studies. J Natl Cancer Inst 1987;78:399-405.

3. Hoon DSB, Korn EL, Cochran AJ. Variations in functional immunocompetence of human tumor-draining lymph nodes. Cancer Res 1987;47:1740-4.

4. Wen DR, Hoon DSB, Chang C, Cochran AJ. Variations in lymphokine generation by individual lymph nodes draining human malignant tumors. Cancer Immunol Immunother 1989;30:277-82.

5. Cochran AJ, Wen DR, Herschman JR. Occult melanoma in lymph nodes detected by antiserum to S-100 protein. Intl J Cancer 1984;34:159-63.

6. Cochran AJ, Wen DR, Morton DL. Occult tumor cells in the lymph nodes of patients with pathological stage I malignant melanoma: an immunohistological study. Am J Surg Pathol 1988;12:612-8.
7. Morton DL, Wen DR, Wong JH, Economou JS, Cagle LA, Storm FK, et al. Technical details of intraoperative lymphatic mapping for early stage melanoma. Arch Surg 1992; 127:392-9.

8. Hoon DS, Bowker RJ, Cochran AJ. Suppressor cell activity in melanoma-draining lymph nodes. Cancer Res 1987;47:152933.

9. Hoon DSB, Bowker R, Cochran AJ. Suppressor cell activity in breast cancer-draining lymph nodes. Surg Res Commun 1990;9:167-76.

10. Farzad Z, Cochran AJ, McBride WH, Grey D, Wong V, Morton DL. Phenotypic changes in lymphocytes of lymph nodes draining human cutaneous melanoma. Cancer Res 1990;50: 3585-8.

11. Farzad Z, McBride WH, Ogbechi H, Asnong-Holthoff C, Morton DL, Cochran AJ. Lymphocytes from lymph nodes at different distances from human melanoma vary in their capacity to inhibit/enhance tumor cell growth in vitro. Melanoma Res 1997;7(Suppl 2):S59-65.

12. Hoon DSB, Irie RF, Cochran AJ. Gangliosides from human melanoma immunomodulate the response of $\mathrm{T}$ cells to interleukin-2. Cell Immunol 1988;111:410-9.

13. Cochran AJ, Hoon DB, Korn EL, Ferraro A, Stene M. Effect of indomethacin on the immunocompetence of human tumordraining lymph nodes [Abstract]. Fed Proc 1985;44:965.

14. Huang R-R, Wen D-R, Guo J, Giuliano A, Turner R, Nguyen M, Cochran AJ. Modulation of paracortical dendritic cells and $\mathrm{T}$ lymphocytes in breast cancer sentinel nodes. Breast J 2000;6:225-32.

15. Hoon DSB, Jung T, Naungayan J, Cochran AJ, Morton DL, McBride WH. Modulation of human macrophage functions by gangliosides. Immunol Lett 1989;20:269-76.

16. Steinman RM, Witmer-Pack M, Inaba K. Dendritic cells: antigen presentation, accessory function and clinical relevance. In: Kamperdijk EWA, Nieuwenhuls P, Hoefsmit ECM, editors. Dendritic cells in fundamental and clinical immunology. New York: Plenum Press; 1993. pp. 1-9.

17. Guesdon JL, Ternynck T, Avrameas S. The use of avidinbiotin interaction in immunoenzymatic techniques. J Histochem Cytochem 1979;27:1131-9.

18. Mickel RA, Kessler DJ, Taylor JMG, Liechtenstein A. Natural killer cell cytotoxicity in the peripheral blood, cervical lymph nodes, and tumors of head and neck patients. Cancer Res 1988;48:5017-22. 